\title{
Incidental or secondary findings: an integrative and patient- inclusive approach to the current debate
}

\author{
Marlies Saelaert $\mathbb{D}^{1} \cdot$ Heidi Mertes ${ }^{2} \cdot$ Elfride De Baere $^{3} \cdot$ Ignaas Devisch $^{4}$
}

Received: 20 December 2017 / Revised: 13 April 2018 / Accepted: 24 May 2018 / Published online: 3 July 2018

(c) European Society of Human Genetics 2018

\begin{abstract}
Incidental or secondary findings (ISFs) in whole exome or whole genome sequencing have been widely debated in recent literature. The American College of Medical Genetics and Genomics' recommendations on diagnostic ISFs have strongly catalyzed the discussion, resulting in worldwide reactions and a variety of international guidelines. This article will outline how propositions on levels of terminology, policy, and underlying values are still internationally criticized and adjusted. Unsolved questions regarding ISFs include a suitable terminology, adequate counseling or informed consent procedures, opt-out possibilities, reporting ISFs to (parents of) minors and values regarding professional duty, patient autonomy, and actionability. These questions will be characterized as intrinsically related and reciprocally maintained and hence, symptomatic, single-level reflections will be marked as ineffective. Instead, a level-integrative approach of the debate that explicitly acknowledges this interaction and considers a balance between internationally significant and case-specific solutions, will be advocated. Second, the inclusion of a patient perspective will be strongly encouraged to complement the professional preponderance in the current debate. The examination of lived patient experiences, a qualitative focus on the subjective meaning of ISFs, and a contextualization of meaning processes will be suggested as specific concretizations. This integrative and inclusive approach aims for a more comprehensive understanding of ISFs, a consideration of all relevant stakeholders' perspective and, ultimately, an effective health-care policy.
\end{abstract}

\section{Introduction}

Incidental findings (IFs) or secondary findings (SFs), being results unrelated to the initial indication for genetic testing, have aroused a vast debate in the literature on whole exome sequencing (WES) or whole genome sequencing (WGS) [1-3]. The initial (2013) recommendations on diagnostic IFs by the American College of Medical Genetics and Genomics (ACMG) have initiated the discussion, while the

Marlies Saelaert

Marlies.Saelaert@ugent.be

1 Department of Family Medicine and Primary Health Care, Ghent University, Ghent, Belgium

2 Department of Philosophy and Moral Sciences, Bioethics Institute Ghent, Ghent University, Ghent, Belgium

3 Center for Medical Genetics Ghent (CMGG), Ghent University and Ghent University Hospital, Ghent, Belgium

4 Department of Family Medicine and Primary Health Care, Ghent University, Ghent, Belgium updated ACMG guidelines of 2015 can be perceived as the start of a second and reoriented debate phase [4, 5]. As a stimulating precedent, the ACMG guidelines have had an international impact, as (explicitly) confirmed in the many statements that were released shortly after them. When comparing these international documents, a diversity in terminology and policy guidelines on incidental or secondary findings (ISFs) can be identified, which has resulted in currently unsolved points of discussion. "ISFs" is used in this article as a working term ad interim, referring to the entirety of both deliberately pursued and accidentally found results that are unrelated to the indication for diagnostic genetic testing, however irrespective of any further specification concerning (clinical) validity or utility, policy or values as suggested by existing literature. Rather than taking a final stance in this debate, this article will explicitly indicate the link between the level of semantic choices and the second level of policy recommendations. Finally, commonly cited (bioethical) values will be integrated as a third level. The elaboration of this threeleveled overview of pertinent discussions on ISFs in current literature will, first, reveal how unsolved problems on one 
particular level affect the overall, international debate and hence, how all problems and levels are intrinsically connected. Subsequently, the lack of a genuine patient perspective will be identified as a second obstacle for the debate. Therefore, a level-integrative approach, which explicitly recognizes the levels' interaction, and a patientinclusive approach will be suggested as necessary steps toward a better understanding of and effective debate on ISFs.

Although the evolution of WES/WGS dissolves the border between diagnostics and both research and screening, this reflection is focused on a diagnostic context, where symptomatic patients enter the health-care system with a specific question [6]. This diagnostic situation is considered to be substantially different from a research, screening or direct-to-consumer context with asymptomatic patients/ participants, where the distinction between primary results and ISFs might be even more complex.

\section{Phase 1: Incidental findings}

Referring to a common phenomenon in medicine, ACMG adopted the terminology of IFs in its initial recommendations of 2013 and defined them as "[...] results that are not related to the indication for ordering the sequencing but that may nonetheless be of medical value or utility [...]" [4]. However, labeling findings as incidental in a context of WES/WGS has been terminologically criticized as paradoxical, because discovering numerous variants is intrinsic to these techniques [7, 8]. Moreover, the characterization of results as incidental has been considered, also by patients, to suggest a sense of insignificance, which is inappropriate in situations of life-saving findings $[9,10]$.

ACMG's policy recommendations revealed a more specific understanding of IFs than the definition suggested. A standard analysis and report of (likely) pathogenic variants (class 5 and class 4 variants that, respectively, affect and probably affect function, including the importance of a contextualized interpretation and the absence of a $100 \%$ certainty regarding pathogenicity and penetrance) in a minimum list of 56 highly-penetrant and medically actionable genes was recommended in any case of diagnostic WES/WGS, irrespective of the indication for testing and of the patient's age and preference [4,11-13]. This implicated the obligatory report of IFs concerning both early- and adult-onset conditions to adults and to (parents of) minors. This recommendation has, again, evoked semantic comments, as it is paradoxical to qualify intentionally sought results as incidental [9]. Therefore, ACMG's parallel reasoning, in which reporting genomic IFs was compared to reporting unexpected radiological anomalies, has also been doubted [4]. While the radiological detection of additional findings cannot be avoided, genomic IFs are oftentimes not inevitable but they are an additional targeted test or they can be covered by use of bioinformatics filters [13, 14]. Also the difference between detecting an actual disorder versus a (future) probability has been regarded as discrediting the parallelism [15]. However, criticism has exceeded the terminological level and the intentional and mandatory analysis and report of IFs have been fundamentally questioned. First, in an explicit reaction to the ACMG recommendations, the Association of Genetic Nurses and Counsellors (AGNC) has stated that intentionally looking for additional results that exceed the indication for a test or consult, is not a routine action in general medical practice [16]. Moreover, the deliberate search for IFs can blur the boundary between diagnostics and screening. This hybridization is, however, not unproblematic, as diagnostics and screening imply different duties, expectations, and values, for both patients/ participants and professionals $[17,18]$. It can also stimulate a trend of medicalization, in which additional screening is a priori considered as beneficial while it can actually result in an overload of (uncertain) information and a group of "patients-in-waiting" [19, 20]. Nonetheless, ACMG seemed to consider this blurring delineation as unproblematic and referred to IFs as "opportunistic screening" [4]. The Presidential Commission for the Study of Bioethical Issues (Bioethics Commission) has questioned this "better safe than sorry" attitude, as opportunistic screening might hold additional risks instead of an actual improvement of care [20]. Second, obligatory reporting results, also against patients' will, violates the general medical practice and policy [19]. Therefore, the Bioethics Commission has upheld the respect for a patient's choice not to be informed about ISFs [20]. Despite its recommendation to report serious and actionable IFs, also the European Society of Human Genetics (ESHG) has stressed how, in general, patients should be able, like in every presymptomatic genetic test setting, to apply and change their preference regarding the disclosure of results [8]. Also a survey among US-based genetic counselors about the ACMG guidelines' implementation confirmed the preference for an opt-out possibility of IFs [21]. Regarding the mandatory report of results about adult-onset conditions to (parents of) minors in particular, the AGNC and others have stated how this practice is incompatible with general pediatric genetic testing [13, 16, 22].

Ultimately, the policy discussion was grounded in a different prioritizing of values. Promoting the active search for IFs, ACMG referred to the professional duty of avoiding harm, both toward patients and their families [4, 11]. Fully respecting this value implicated informing (parents of) minors about IFs concerning adult-onset conditions, as it might be the only opportunity to avoid serious future 
morbidity in the child's relatives [4, 23]. This way, ACMG's exception on declining presymptomatic tests in minors for adult-onset conditions was justified by a familywide conception of health benefits [23, 24]. In an explicit reflection on the ACMG and ESHG guidelines, also the Canadian College of Medical Geneticists (CCMG) has stated how this practice, in case of unintentional IFs, might be opportune when it can avoid serious medical harm and when explicitly requested by the parents [25]. This point of view was shared by laboratory professionals who theoretically did not differentiate between reporting IFs to adults or (parents of) minors and by clinical geneticists and genetic researchers who have considered the return of adult-onset results in minors as possibly opportune [26-28]. Also parents considered the possibility of receiving these results as a positive opportunity for additional information about themselves [29]. In contrast to the obligatory report of IFs, critics have stressed the professional duty to respect the medical choice of (parents of) patients, including the wish not to be informed, and this in respect of the fundamental value of (future) patient autonomy [13, 19, 22, 30]. Therefore, in a reply to the ACMG recommendations, the Public Health Genetics (PHG) Foundation has stated that denying a patient's consent in opportunistic screening is an unethical practice [31]. Also a US-based expert forum on the ACMG recommendations stressed the professional duty of respecting patients' right not to be informed [32]. ACMG recognized how its recommendations collided with ethical values but explicitly confirmed that, in this case, the duty to avoid harm exceeded the value of autonomy [4].

Possibly, ACMG's position was motivated by the specific US health-care context and by fear of legal consequences for not reporting "all available information". However, it has been argued that the ACMG recommendations even enlarge liability risks, as professionals might be sued, e.g., for delayed disclosure or failure of reevaluating sequence data, or, on the other hand, for needless or harmful follow-up for IFs [33]. Moreover, the frequently divergent claims of other US-based professionals and policy groups threaten the absolute weight of the liability concern [13, 20, 22]. Finally, a European versus US geographical background turned out to be of no significant impact on professionals' attitude toward the return of IFs $[34,35]$.

\section{Phase 2: Secondary findings}

In response to the terminological critique on IFs, alternatives such as "unsolicited", "unanticipated", or "unexpected" findings, or "secondary variants" have been suggested [7-9, 36]. The Bioethics Commission has chosen a multiple vocabulary and has discerned anticipatable and unanticipatable IFs, SFs and discovery findings [20]. Partly in line with this terminology, ACMG has revised its vocabulary from "incidental" to "secondary" findings, as this term acknowledges the intentional search for additional pathogenic variants [5, 12, 37]. However, none of all the terminological suggestions have remained free of objections, as they might deny a professional's competence to anticipate specific variants, neglect different expectations of different stakeholders, or overlook cases where no primary result has been found $[2,9,20,38]$.

The adjustment of ACMG's vocabulary has coincided with a major change in its recommended policy as a possibility of opting out of SFs has been offered to patients [5]. Nonetheless, the idea of, even voluntary, opportunistic screening is incompatible with the intrinsic questioning of SFs by some policy groups. As part of the professional duty of non-maleficence, the Bioethics Commission has advocated "therapeutic parsimony", being a selectivity in chosen tests or interventions, and "diagnostic elegance", being a limitation of potential diagnoses. Therefore, in general, targeted testing is considered as more suitable, as it inhibits the possible downstream of medical, financial, and psychological procedures after identifying ISFs [20]. In order to avoid ISFs and their high-cost impact on patients, families, and society, the CCMG, ESHG, and EuroGentest have also recommended, in explicit reflection on previous guidelines such as those of ACMG, an initial targeted testing and a justification of WES/WGS in terms of necessity and proportionality $[6,8,25]$.

ACMG's adjusted possibility for opting out of SFs has reinstated the value of patient autonomy. However, on an international level, the absolute versus relative weight of this value and its application to IFs, SFs, or both are unclear. ACMG has made no explicit notion of cases where opt-out is or should be impossible for SFs and also the AGNC has defended autonomy as "the heart of genetic counseling practice" in opportunistic screening [5, 16, 37]. The Bioethics Commission has, despite its claim for an opt-out possibility, only granted a relative weight to autonomy: when ISFs are clinically significant, of serious health importance and actionable, a "prudent professional judgment" should be made and the patient's opt-out choice should be respected "to the extent consistent with the clinician's fiduciary duty" [20]. Also the ESHG and the PHG Foundation have affirmed how the right not to know IFs does not always exceed the professional duties of beneficence and non-maleficence, e.g., when the information might be actionable and relevant for patients themselves and/or their (future) family $[8,31]$. 


\section{Medical actionability}

In both ACMG's initial and updated recommendations, medical actionability, being the possibility of an improved clinical management by medical treatment or prevention, has been displayed as a fundamental value [3, 39]. In 2016, a semiquantitative metric to score genes regarding their medical actionability has been elaborated, using the criteria of severity and likelihood of disease outcome, efficacy and acceptability of the intervention, and the knowledge-base regarding the previous four criteria [40]. No reference is made to the possible disease outcomes for a patient's family or (future) offspring, neither to interventions as patientperformed actions such as lifestyle changes or reproductive choices. Consequently, actionability is conceived as the possibility of strictly medical, professionally performed interventions toward the actual patient. In accordance with this metric, ACMG has updated 5 genes on its initial list, resulting in a minimum list of 59 genes $[12,37]$. This semiquantitative definition of medical actionability has been widely criticized and, as a first comment, it has been mentioned, e.g., by the Bioethics Commission, how difficult it can be to exactly assess the true medical value and actionability of ISFs at the moment of discovery [20]. Variants can have an unknown pathogenicity when discovered in asymptomatic persons and their significance depends on further investigations, of both patients and their family [1, 13, 19]. Hofmann [41] even claims how the ACMG list mainly consists of findings of uncertain significance and of results which lack accuracy and actionability. As a second critique, the required professional knowledge to assess a gene's medical actionability and the generalization of a professional criterion into a universal value have been blamed to result in a degree of paternalism that is incongruent with the current, pluralistic, and patientcentered society $[14,39]$. Finally, it should be noticed how ACMG's use of this semiquantitative metric contradicts its previous argument of a family-wide health interest in case of ISFs concerning adult-onset conditions in minors.

In order to deny the monopoly of a strictly medical and professional actionability, a wide spectrum of alternatives has been suggested. Moret et al. [42] assert a concept of actionability that discerns well-established medical actions, patient-initiated health-related actions, and patient-initiated decisions exceeding health, such as reproductive choices. Stivers and Timmermans consider actionability as an interactional value that is created in the relation between (parents of minor) patients and clinicians. Even if genetic results do not change (parents of minor) patients' (medical) actions, they can be actionable in various meaningful ways, e.g., by facilitating specific services (e.g., educational services for disabled children) or by changing psychological experiences or reproductive choices. Hence, actionability is not an objective, medical criterion but is determined by (parents of minor) patients' personal, social, reproductive, etc. context [43]. Concepts of actionability that surpass a medical focus acknowledge the personal utility of genetic information and consider warning at-risk relatives and adjusting behavior or reproductive choices as valuable actions, a perception also shared by the Bioethics Commission [14, 20, 30, 39]. This extended actionability approach also recognizes an intrinsic value of genetic knowledge per se, irrespective of any practical use. Various stakeholders (professionals, patients, research participants, and the general public) have supported this idea and have preferred to return or receive "all results", regardless of their actionability [36, 39, 44]. In line with this enlarged concept of actionability, the PHG Foundation's list of disclosure criteria for ISFs includes, e.g., the age and general condition of the patient, which suggests a more diverse spectrum of reportable results that exceeds medical actionability [31].

\section{A level-integrative and patient-inclusive approach}

Despite ACMG's adjustment of its vocabulary, recommended policy, and prioritized value, international disagreement has remained (Fig. 1). On terminology level, alternative terms for ISFs are still suggested and used inconsistently, keeping consensus out of reach [7]. It also generates a vagueness in policy publications on whether they actually apply to IFs, SFs, or both. On policy level, adequate counseling and informed consent procedures are a general problem regarding ISFs. The evolution toward WES/WGS, providing an enormous amount of information, challenges which information and counseling are required to realize a truly informed consent and a satisfactory understanding of (additional) results [44, 45]. Professionals and sometimes (parents of minor) patients themselves have expressed their concern about a limited understanding of WES/WGS, an ignorance that might partially explain people's desire to receive a very broad range of (additional) results $[29,44,46]$. More fundamentally, the feasibility and effectiveness of traditional pre-test procedures in which a large amount of complex information is provided, have been queried [45]. To avoid an overload of information and to realize an enhanced understanding, alternative consent procedures have been elaborated, in which binning systems, which (partially) allow (parents of minor) patients to choose which categories of possible results to receive, have been frequently suggested. Berg et al. already elaborated a categorical framework for IFs, where "bin 1 findings", consisting of (likely) pathogenic variants in medically actionable genes, were recommended to report. The return of (likely) pathogenic variants in clinically non-actionable 
Fig. 1 Two-phased, threeleveled debate on ISFs

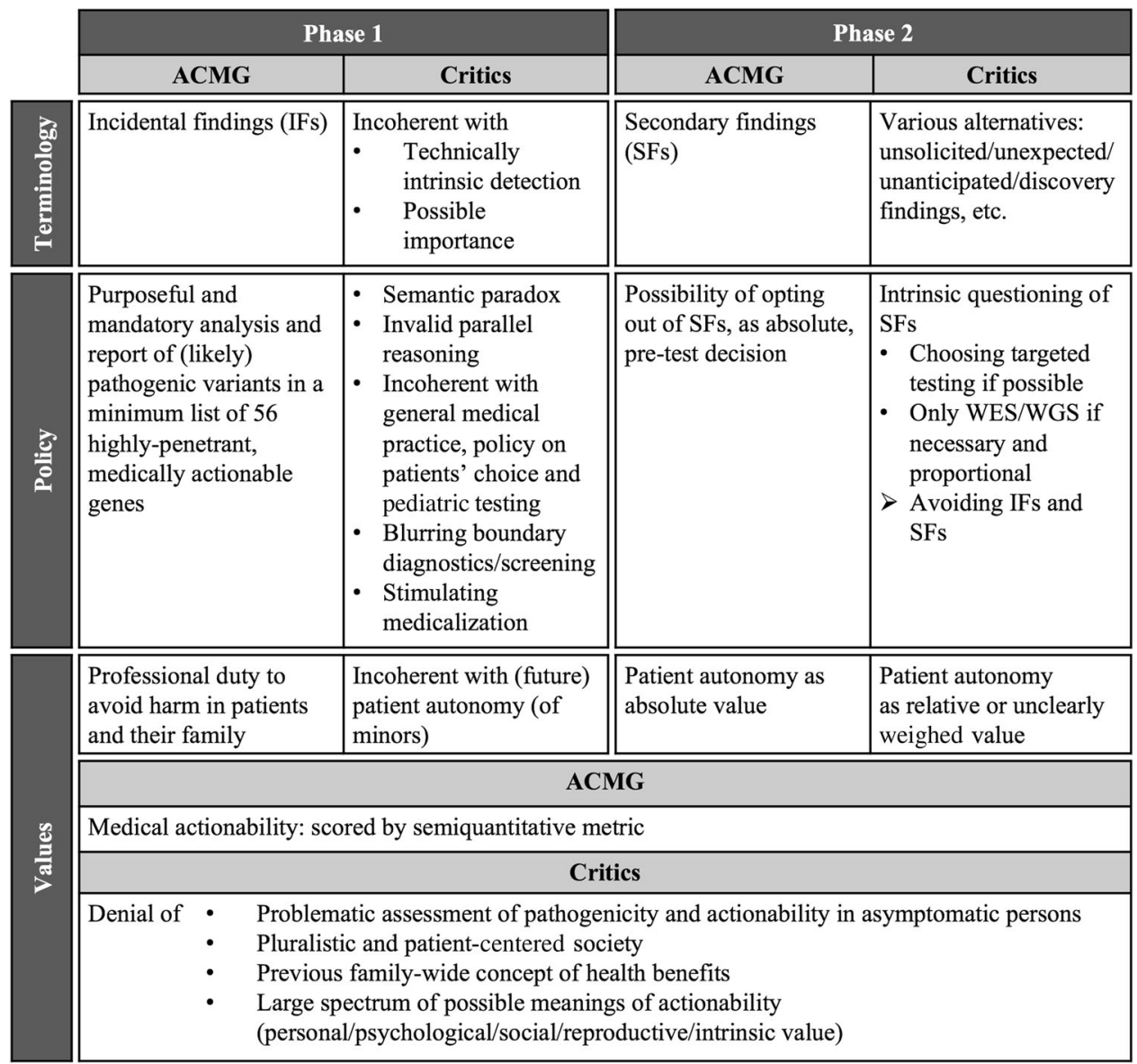

genes depended on a shared decision-making between the (parents of the) patient and the professional, whereas genes and variants of unknown significance should never be reported, as their informative value is unclear [47, 48]. Elaborating on such binned systems, tiered/layered procedures of consent have been suggested, where a default package of necessary information is presented to all (parents of minor) patients, while more detailed information is only selectively provided, depending on specific information needs and result preferences $[49,50]$. Despite the usefulness of these systems, an effective integration in clinical practice is still impeded. First, some categories or bins lack an exact definition, with the concept of actionability as specifically problematic [42]. Second, professionals have disagreed if a patient's age can affect the return of specific categories of results, an issue related to the return of ISFs regarding adultonset conditions in minors [51, 52]. Third, despite these binned, tiered, or layered consent procedures, it has been suggested that, in general, too much focus has been put on the informational aspect of counseling and that instead, more attention should be paid to its interactional, collaborative, and ethical nature [45]. Besides this general challenge of an adequate counseling process for ISFs, also the policy on opting out requires further clarification. Related to the terminological vagueness, it is, e.g., unclear whether ACMG's and AGNC's opt-out possibility only applies to SFs or also to IFs, a question also linked to the undetermined weight of patient autonomy. A similar vagueness occurs in the Bioethics Commission's recommendation on balancing patients' possible opt-out preference versus professional duties regarding both IFs and SFs. The ESHG and EuroGentest have plead for a clear optin and opt-out protocol regarding ISFs, both for adult and minor testing, but again, no specificities have been given $[6,8]$. The policy of avoiding ISFs and intentionally covering results by bioinformatics filters raises ethical questions because, even though these results are masked for the professional eye, they still exist. It is unclear if this mask actually eliminates the professional duty to avoid harm, an issue also referred to by the Bioethics Commission's claim that the fiduciary duty does not allow professionals to filter additional results exclusively in order to avoid responsibility $[17,20]$. Also the practice of reporting ISFs (especially those related to adult-onset conditions) in minor testing still raises doubts, on both policy and value levels. An interest has been shown in results that are not (yet) 
relevant for tested (minor) patients themselves, hence it is debatable if (minor) patients' results can be used for others' possible benefit or if this is an unacceptable instrumentalization [24]. A thorough reflection is needed on values such as (future) autonomy and the right not to know, and whether these values, in the context of current genetics, still have their traditional meaning or if they are in need of a conceptual update.

Regarding the value of actionability, a feasible concept should be elaborated that can effectively guide practice and policy. Berg's semiquantitative metric for medical actionability seems a straightforward procedure to classify ISFs, but a gene's correct categorization can be difficult [14, 37, 53]. For example, scoring a gene on the severity of its outcome or scoring an intervention on efficacy is ambiguous when the gene is associated with multiple outcomes or when different interventions are available. It is undecided in these cases whether the most severe or the most likely outcome should be scored or whether very radical interventions should also be considered [53]. Moreover, the likelihood of a possible disease outcome and the efficacy and acceptability of an intervention are partly determined by (the parents of) a patient's characteristics and context, an argument also recognized by Berg himself [40]. These pitfalls of a rigorous metric show the difficulty to measure actionability by merely medical criteria and the need to find common ground for patients, parents, and professionals on the wide spectrum between a strictly medical interpretation and more subjective and contextualized interpretations.

The aforementioned problems regarding ISFs clearly demonstrate a reciprocal interdependence and hence a strong unity of terminology, policy, and values. The terminological vagueness is reflected in ambiguous guidelines, while the unsettled meaning and weight of ethical values fail to support effective policy recommendations [31]. Denying this constant interaction results in limited answers to only partial problems and inhibits an adequate approach of the overall debate. Therefore, as a first recommended approach to the debate, an absolute integration of all levels in every consideration of ISFs is strongly advocated and a withdrawal of symptomatic questions that neglect this interaction is an absolute necessity. This level-integrative approach does not demand the instant and simultaneous solution of all aforementioned problems, nor the pursuit of an international consensus on all levels. However, the debate on ISFs should acknowledge how terminological choices and policy recommendations lack solidity when the underlying levels (of policy and/or values), to which they always (implicitly) refer, are disregarded. This lacking solidity inhibits an effective implementation of levelspecific decisions, which can result in a diversified practice, an inequity in access of care and a suboptimal organization of care. On an international level, unsolid and largely heterogeneous answers on terminology, policy, and value problems can undermine the guidance and efficacy of these answers and erode the significance of important principles and values [35]. Nonetheless, pertinent ethical, legal, and societal differences exist, e.g., between the United States and Europe, which devaluates the aim of global guidelines $[7,35]$. Moreover, the casuistry of a patient's specific context and a professional's particular judgment impede the idea of a strict uniformity [26]. A level-integrative approach should consider this balance between the pursuit of internationally significant answers and the necessity of case-bycase solutions on all levels of the ISFs debate.

This level-integrative approach of the debate will still lack important information, as ISFs have been mainly considered by (boards of) professionals. The experiences of (parents of minor) patients who encounter the possibility of ISFs are largely unexplored, which further erodes the debate. Hence, as a second recommended approach, an inclusion of the perspective of actual end-users is advocated, as a necessary complement to the current professional, top-down approach. Numerous publications have stressed the importance of the patient perspective on ISFs but these calls have stayed too vague and have lacked actual realization [4, 13, 15, 20, 37].

Therefore, as a first concretization of the inclusion of a patient perspective, lived experiences of (parents of) a real patient population should be pursued. Current research frequently suffers from a hypothetical bias by interrogating people who have to simulate a different role (e.g., of a patient, parent, or family member) or a different medical situation (e.g., having a diagnostic question or being genetically tested). Hypothetical discussions, however, can be very different from lived experiences, which is demonstrated in the increased selectivity in preferred ISFs by patients with an actual experience of illness and genetic testing [11, 44].

Second, an explicit focus on (possible) ISFs' meaning and significance is suggested. Instead of (quantitatively) measuring (parents of minor) patients' evaluation of professional classifications or recommendations, a qualitative insight in patients' or parents' subjective meaning of ISFs is recommended. This objective is supported by the observation that professionally determined categories or bins for ISFs, e.g., (medically) actionable results, do not necessarily correspond with patients' or parents' perception [44, 48]. This discrepancy in terminology and underlying values between important stakeholders holds the risk to make policy instruments ineffective. It is fully acknowledged that more inclusive and subjective perspectives on actionability and other values challenge the counseling process and return of results. Nonetheless, it is doubtful whether this is an acceptable excuse to deny personally useful information to (parents of minor) patients. 
Finally, the specific context of a patient's subjective perspective on ISFs should be emphasized. Patient-related factors such as patients' or parents' family history, social support, primary condition, and previous experiences with genetic counseling can all mediate the meaning of ISFs $[20,39]$. This suggests that ISFs' significance is not constructed by single-dimension criteria (such as pathogenicity or actionability) but by a complex interaction of multiple, contextualized criteria. Therefore, (parents of minor) patients might also favor more dynamic or staged consent procedures, in which the validity of personal preferences is not limited to a single pre-test moment. Having the possibility to give consent at several times, e.g., prior to testing, prior to receiving (specifically preferred) results, and prior to updates about these results, can allow to weigh values (e.g., actionability, professional duty, personal autonomy, etc.) differently in different situations and to make specifically contextualized decisions. It also allows that information and counseling are (repeatedly) provided and adjusted to evolving scientific knowledge [48, 50]. Finally, contextualized and dynamic policy procedures can stimulate or necessitate a diversified terminology to cover the different meanings ascribed to ISFs, which, again, confirms the intrinsic interaction of terminology, policy, and values regarding ISFs [38].

\section{General conclusion}

Despite the international guidelines on ISFs, a complex interaction of various problems still confiscates the debate, which impedes an adequate and effective implementation of the promising techniques of WES/WGS. The diagnostic possibilities of these techniques are captivating but the required knowledge to manage all results and additional information, is challenging. A level-integrative and patientinclusive approach to the debate on ISFs pursues a more comprehensive understanding of ISFs. It explicitly recognizes, first, the intrinsic interaction between the different levels of the debate and, second, the importance of a lived, subjective, and contextualized patient perspective. Ultimately, the integration and alignment of terminology, policy, and values, and the inclusion of all relevant stakeholders will support the realization of an effective, well-grounded practice regarding ISFs.

Funding Special research fund: Concerted Research Actions (BOFGOA) BOF15/GOA/011; research foundation: Flanders (FWO) $1802215 \mathrm{~N}$; research foundation: Flanders (FWO) 1205415N.

\section{Compliance with ethical standards}

Conflict of interest The authors declare that they have no conflict of interest.

\section{References}

1. Crawford G, Foulds N, Fenwick A, Hallowell N, Lucassen A. Genetic medicine and incidental findings: it is more complicated than deciding whether to disclose or not. Genet Med. 2013;15:896-9.

2. Shkedi-Rafid S, Dheensa S, Crawford G, Fenwick A, Lucassen A. Defining and managing incidental findings in genetic and genomic practice. J Med Genet. 2014;51:715-23.

3. Bennette CS, Trinidad SB, Fullerton SM, et al. Return of incidental findings in genomic medicine: measuring what patients value-development of an instrument to measure preferences for information from next-generation testing (IMPRINT). Genet Med. 2013;15:873-81.

4. Green RC, Berg JS, Grody WW, et al. ACMG recommendations for reporting of incidental findings in clinical exome and genome sequencing. Genet Med. 2013;15:565-74.

5. ACMG Board of Directors. ACMG policy statement: updated recommendations regarding analysis and reporting of secondary findings in clinical genome-scale sequencing. Genet Med. 2015;17:68-69. https://doi.org/10.1038/gim.2014.151.

6. Matthijs G, Souche E, Alders M, et al. Guidelines for diagnostic next-generation sequencing. Eur J Hum Genet. 2016;24:2-5.

7. Hehir-Kwa JY, Claustres M, Hastings RJ, et al. Towards a European consensus for reporting incidental findings during clinical NGS testing. Eur J Hum Genet. 2015;23:1601-6.

8. van El CG, Cornel MC, Borry P, et al. Whole-genome sequencing in health care Recommendations of the European Society of Human Genetics. Eur J Hum Genet. 2013;21:580-4.

9. Christenhusz GM, Devriendt K, Dierickx K. Secondary variantsin defense of a more fitting term in the incidental findings debate. Eur J Hum Genet. 2013;21:1331-4.

10. Tan NN, Amendola LM, O'Daniel JM, et al. Is "incidental finding" the best term?: a study of patients' preferences. Genet Med. 2017;19:176-81.

11. Regier DA, Peacock SJ, Pataky R, et al. Societal preferences for the return of incidental findings from clinical genomic sequencing: a discrete-choice experiment. Can Med Assoc J. 2015;187:E190-7.

12. Scheuner MT, Peredo J, Benkendorf J, et al. Reporting genomic secondary findings: ACMG members weigh. Genet Med. 2015;17:27-35.

13. Burke W, Antommaria AHM, Bennett R, et al. Recommendations for returning genomic incidental findings? We need to talk! Genet Med. 2013;15:854-9.

14. May T. On the justifiability of ACMG recommendations for reporting of incidental findings in clinical exome and genome sequencing. J Law Med Ethics. 2015;43:134-42.

15. Townsend A, Adam S, Birch PH, Friedman JM. Paternalism and the ACMG recommendations on genomic incidental findings: patients seen but not heard. Genet Med. 2013;15:751-2.

16. Middleton A, Patch C, Wiggins J, et al. Position statement on opportunistic genomic screening from the Association of Genetic Nurses and Counsellors (UK and Ireland). Eur J Hum Genet. 2014;22:955-6.

17. Berg JS, Amendola LM, Eng C, et al. Processes and preliminary outputs for identification of actionable genes as incidental findings in genomic sequence data in the Clinical Sequencing Exploratory Research Consortium. Genet Med. 2014;16:203.

18. Dheensa S, Shkedi-Rafid S, Crawford G, Bertier G, Schonstein L, Lucassen A. Management of incidental findings in clinical genomic sequencing studies. In: eLS. John Wiley \& Sons, Ltd: Chichester. https://doi.org/10.1002/9780470015902.a0025838. January 2016.

19. Ross LF, Rothstein MA, Clayton EW. Mandatory extended searches in all genome sequencing "incidental findings," patient autonomy, and shared decision making. JAMA. 2013;310:367-8. 
20. Presidential Commission for the Study of Bioethical Issues. Anticipate and communicate: ethical management of incidental and secondary findings in the clinical, research, and direct-toconsumer contexts. 2013. http://bioethics.gov/sites/default/files/ FINALAnticipateCommunicate_PCSBI_0.pdf. Accessed June 2017.

21. Smith LA, Douglas J, Braxton AA, Kramer K. Reporting incidental findings in clinical whole exome sequencing: incorporation of the 2013 ACMG recommendations into current practices of genetic counseling. J Genet Couns. 2015;24:654-62.

22. Allyse M, Michie M. Not-so-incidental findings: the ACMG recommendations on the reporting of incidental findings in clinical whole genome and whole exome sequencing. Trends Biotechnol. 2013;31:439-41.

23. American College of Medical Genetics and Genomics. Incidental findings in clinical genomics: a clarification. Genet Med. 2013; 15:664-6.

24. Christenhusz GM, Devriendt K, Van Esch H, Dierickx K. Focus group discussions on secondary variants and next-generation sequencing technologies. Eur J Med Genet. 2015;58:249-57.

25. Boycott K, Hartley T, Adam S, et al. The clinical application of genome-wide sequencing for monogenic diseases in Canada: position statement of the Canadian College of Medical Geneticists. J Med Genet. 2015;52:431-7.

26. Vears DF, Senecal K, Borry P. Reporting practices for unsolicited and secondary findings from next-generation sequencing technologies: perspectives of laboratory personnel. Hum Mutat. 2017;38:905-11.

27. Klitzman R, Appelbaum PS, Fyer A, et al. Researchers' views on return of incidental genomic research results: qualitative and quantitative findings. Genet Med. 2013;15:888-95.

28. Lemke AA, Bick D, Dimmock D, Simpson P, Veith R. Perspectives of clinical genetics professionals toward genome sequencing and incidental findings: a survey study. Clin Genet. 2013;84: 230-6.

29. Krabbenborg L, Schieving J, Kleefstra T, et al. Evaluating a counselling strategy for diagnostic WES in paediatric neurology: an exploration of parents' information and communication needs. Clin Genet. 2016;89:244-50.

30. Christenhusz GM, Devriendt K, Dierickx K. To tell or not to tell? A systematic review of ethical reflections on incidental findings arising in genetics contexts. Eur J Hum Genet. 2013;21:248-55.

31. PHG Foundation. Managing incidental and pertinent findings from WGS in the 100,000 Genomes Project. Cambridge: 2013. ISBN 978-1-907198-12-0; http://www.phgfoundation.org/ documents/326_1369298828.pdf

32. Johnson KJ, Gehlert S. Return of results from genomic sequencing: a policy discussion of secondary findings for cancer predisposition. J Cancer Policy. 2014;2:75-80.

33. Evans BJ. Minimizing liability risks under the ACMG recommendations for reporting incidental findings in clinical exome and genome sequencing. Genet Med. 2013;15:915-20.

34. Middleton A, Morley KI, Bragin E, et al. Attitudes of nearly 7000 health professionals, genomic researchers and publics toward the return of incidental results from sequencing research. Eur J Hum Genet. 2016;24:21-9.

35. Gourna EG, Armstrong N, Wallace SE. Compare and contrast: a cross-national study across UK, USA and Greek experts regarding return of incidental findings from clinical sequencing. Eur J Hum Genet. 2016;24:344-9.

36. Christenhusz GM, Devriendt K, Peeters H, Van Esch H, Dierickx $\mathrm{K}$. The communication of secondary variants: interviews with parents whose children have undergone array-CGH testing. Clin Genet. 2014;86:207-16.

37. Kalia SS, Adelman K, Bale SJ, et al. Recommendations for reporting of secondary findings in clinical exome and genome sequencing, 2016 update (ACMG SFv2.0): a policy statement of the American College of Medical Genetics and Genomics. Genet Med. 2017;19:249-55.

38. Crawford G, Fenwick A, Lucassen A. A more fitting term in the incidental findings debate: one term does not fit all situations. Eur J Hum Genet. 2014;22:957.

39. Christenhusz GM, Devriendt K, Van Esch H, Dierickx K. Ethical signposts for clinical geneticists in secondary variant and incidental finding disclosure discussions. Med Health Care Philos. 2015;18:361-70.

40. Berg JS, Foreman AKM, O'Daniel JM, et al. A semiquantitative metric for evaluating clinical actionability of incidental or secondary findings from genome-scale sequencing. Genet Med. 2016;18:467-75.

41. Hofmann B. Incidental findings of uncertain significance: to know or not to know-that is not the question. BMC Med Ethics. 2016;17:9.

42. Moret C, Mauron A, Fokstuen S, Makrythanasis P, Hurst SA. Defining categories of actionability for secondary findings in nextgeneration sequencing. J Med Ethics. 2017;43:346-9.

43. Stivers T, Timmermans $\mathrm{S}$. The actionability of exome sequencing testing results. Sociol Health Ill. 2017;39:1542-56.

44. Mackley MP, Fletcher B, Parker M, Watkins H, Ormondroyd E. Stakeholder views on secondary findings in whole-genome and whole-exome sequencing: a systematic review of quantitative and qualitative studies. Genet Med. 2016;19:283-93.

45. Samuel GN, Dheensa S, Farsides B, Fenwick A, Lucassen A. Healthcare professionals' and patients' perspectives on consent to clinical genetic testing: moving towards a more relational approach. BMC Med Ethics. 2017;18:11.

46. Rigter T, van Aart CJA, Elting MW, Waisfisz Q, Cornel MC, Henneman L. Informed consent for exome sequencing in diagnostics: exploring first experiences and views of professionals and patients. Clin Genet. 2014;85:417-22.

47. Berg JS, Khoury MJ, Evans JP. Deploying whole genome sequencing in clinical practice and public health: meeting the challenge one bin at a time. Genet Med. 2011;13:499-504.

48. Roche MI, Berg JS. Incidental findings with genomic testing: implications for genetic counseling practice. Curr Genet Med Rep. 2015;3:166-76.

49. Bradbury AR, Patrick-Miller L, Long J, et al. Development of a tiered and binned genetic counseling model for informed consent in the era of multiplex testing for cancer susceptibility. Genet Med. 2015;17:485-92.

50. Bunnik EM, Janssens A, Schermer MHN. A tiered-layered-staged model for informed consent in personal genome testing. Eur $\mathrm{J}$ Hum Genet. 2013;21:596-601.

51. Green RC, Berg JS, Berry GT, et al. Exploring concordance and discordance for return of incidental findings from clinical sequencing. Genet Med. 2012;14:405-10.

52. Rigter T, Henneman L, Kristoffersson U, et al. Reflecting on earlier experiences with unsolicited findings: points to consider for next-generation sequencing and informed consent in diagnostics. Hum Mutat. 2013;34:1322-8.

53. Lazaro-Munoz G, Conley JM, Davis AM, Prince AER, Cadigan RJ. Which results to return: subjective judgments in selecting medically actionable genes. Genet Test Mol Biomark. 2017; 21:184-94. 